\title{
BMJ Open Development and validation of a patient-reported outcome tool to assess cancer-related financial toxicity in Italy: a protocol
}

\author{
Silvia Riva, ${ }^{1,2}$ Jane Bryce, ${ }^{3,4,5}$ Francesco De Lorenzo, ${ }^{6,7}$ Laura Del Campo, ${ }^{8}$ \\ Massimo Di Maio, ${ }^{9}$ Fabio Efficace, ${ }^{10}$ Luciano Frontini, ${ }^{11}$ Diana Giannarelli, ${ }^{12}$ \\ Lara Gitto, ${ }^{13}$ Elisabetta lannelli, ${ }^{8}$ Claudio Jommi, ${ }^{14}$ Vincenzo Montesarchio, ${ }^{15}$ \\ Francesca Traclò, ${ }^{6}$ Concetta Maria Vaccaro, ${ }^{16}$ Ciro Gallo, ${ }^{17}$ Francesco Perrone ${ }^{3}$
}

To cite: Riva S, Bryce J, De Lorenzo F, et al. Development and validation of a patientreported outcome tool to assess cancer-related financial toxicity in Italy: a protocol. BMJ Open 2019;9:e031485. doi:10.1136/ bmjopen-2019-031485

- Prepublication history for this paper is available online. To view please visit the journal online (http://dx.doi.org/10. 1136/bmjopen-2019-031485)

Received 07 May 2019 Revised 06 August 2019 Accepted 28 August 2019

Check for updates

(C) Author(s) (or their employer(s)) 2019. Re-use permitted under CC BY-NC. No commercial re-use. See rights and permissions. Published by BMJ.

For numbered affiliations see end of article.

Correspondence to Dr Francesco Perrone; f.perrone@istitutotumori.na.it

\section{ABSTRACT}

Introduction Financial toxicity (FT) is a well-recognised problem in oncology. US-based studies have shown that: (a) cancer patients have a 2.7 times risk of bankruptcy; (b) patients who declare bankruptcy have a $79 \%$ greater hazard of death; (c) financial burden significantly impairs quality of life (QoL) and (d) reduces compliance and adherence to treatment prescriptions. The aim of the project is to develop and validate a patient-reportedoutcome (PRO) measure to assess FT of cancer patients in Italy, where, despite the universal health coverage provided by the National Health Service, FT is an emerging issue.

Methods and analysis Our hypothesis is that a specific FT measure, which considers the relevant sociocultural context and healthcare system, would allow us to understand the main determinants of cancer-related FT in Italy, in order to address and reduce these factors. According to the International Society for Pharmacoeconomics and Outcomes Research guidelines on PR0s, the project will include the following steps: (1) concept elicitation (from focus groups with patients and caregivers; literature; oncologists; nurses) and analysis, creating a coding library; (2) item generation (using a format that includes a question and a response on a 4-point Likert scale) and analysis through patients' cognitive interviews of item importance within different coding categories to produce the draft instrument; (3) factor analysis and internal validation (with Cronbach's alpha and test-retest for reliability) to produce the final instrument; (4) external validation with QoL anchors and depression scales. The use of the FT measure in prospective trials is also planned.

Ethics and dissemination The protocol is approved by the ethical committees of all the participating centres. The project will tentatively produce a validated tool by the spring 2021. The project might also represent a model and the basis for future cooperation with other European countries, with different healthcare systems and socioeconomic conditions.

Trial registration number NCT03473379.

\section{Strengths and limitations of this study}

- Previous research data support that financial problems affect the outcome of cancer patients in Italy, and despite an Italian healthcare system grounded on universal coverage, financial constraints may possibly affect cancer patient access to healthcare goods and services in the future.

- The way healthcare and welfare systems are structured impacts financial toxicity (FT) suffered by cancer patients, and instruments for describing and measuring FT need to be developed taking healthcare and welfare context into account.

- This protocol applies a widely accepted methodology for the development of patient reported outcome measures.

- The rational of this protocol is that an instrument measuring cancer-related FT may be conducive to corrective policies that might ultimately benefit patients

- For the same reasons that favour the development of a local instrument rather than the application of an existing tool developed elsewhere, one intrinsic limitation is that the instrument being developed will be properly applicable to Italian patients only.

\section{INTRODUCTION}

Financial problems are a matter of global attention in oncology, focusing on two levels of financial difficulties: third payer and individual patient. Payers are generally affected by the rising cost of anticancer drugs. ${ }^{12}$ At the patient level, in the USA, co-payment is becoming unaffordable and, as a consequence, cancer patients have higher likelihood to file for bankruptcy than the general population. ${ }^{3}$ In addition, financial difficulties have been associated with lower patient satisfaction, ${ }^{4}$ worse compliance, ${ }^{5}$ worse quality of life $(\mathrm{QoL})^{6-8}$ and worse survival. ${ }^{9}$ Particularly, Ramsey and collaborators have shown that 
US cancer patients who declared bankruptcy have a HR of death of 1.79 (95\% CI 1.64 to 1.96) as compared with a population of cancer patients who did not file for bankruptcy and were matched in a propensity score analysis. ${ }^{9}$ The problem is being reported in other countries and with varied types of healthcare systems. ${ }^{10-13}$

The need for specific tools to address this problem has been acknowledged in the scientific community, and in the USA a patient-reported-outcome (PRO) instrument, called 'COST', has been recently proposed for measuring financial distress. ${ }^{14}$ COST is a questionnaire composed of 11 items addressing psychological response to financial problems and coping behaviours. This questionnaire does not address material conditions that cause financial problems, and is highly specific for US context, where cancer diagnosis translates into an excess of out-of-pocket expenses. ${ }^{15}$ COST has been validated in some clinical settings, for example, in a series of 100 multiple myeloma patients after at least 3 months of medical treatment, in a series of 233 patients undergoing chemotherapy for solid tumours, and in a series of 191 Japanese patients receiving chemotherapy for a solid tumour for at least 2 months. ${ }^{16-18}$ However, this questionnaire might not be sensitive to relevant issues in health systems where co-payment for anticancer drugs and other healthcare costs is not required.

On this ground, and cognizant of the challenges of cross-cultural applicability to the Italian cancer patient population, we initiated the development of a country specific tool to ensure highest possible content validity. In Italy, we have recently published that financial difficulties exist and negatively affect the outcomes of cancer patients undergoing anticancer treatment, in terms of both QoL and life expectancy. ${ }^{19}$ We used the European Organization for Research and Treatment of Cancer (EORTC) QLQ-C30 questionnaire in several clinical trials of treatment of solid tumours, promoted by the National Cancer Institute of Napoli during the last two decades. Therefore, taking advantage of the existing databases, we performed a pooled analysis of 16 trials including 3670 patients, with the aims of exploring the relevance of financial difficulties in the Italian public healthcare system. We analysed question 28 of the EORTC QLQ-C30 asking: "During the last week, has your physical condition or medical treatment caused you financial difficulties?", with four possible response categories, from $1=$ 'not at all' to $4=$ 'very much'. At baseline, $26 \%$ of the 3670 study patients reported a financial burden (response $\geq 2$ ) that was associated with a higher chance of worsening global QoL response (OR 1.35, 95\% CI 1.08 to $1.70, \mathrm{p}=0.009$ ). During treatment, out of 2735 patients who completed subsequent questionnaires, $616(22.5 \%)$ developed financial toxicity (FT; worsening response as compared with the baseline) that was associated with a statistically significant higher risk of death (HR 1.20; 95\% CI 1.05 to 1.37, $\mathrm{p}=0.007){ }^{19}$

The hypothesis underlying this project is that a specific instrument might help to describe and understand determinants and effects of cancer-related FT in Italy and that some of these determinants may be modified, ultimately improving prognosis of cancer patients.

Therefore, the aim is to develop and validate a PRO instrument to describe and measure FT of Italian patients on cancer treatment, hereby described as 'Patient Reported Outcome for FIghting Financial Toxicity of cancer' (PROFFIT).

The impact of this project might be relevant for patients' prognosis if we were able to understand how to intervene on FT, through adequate policies, because the size of the prognostic impact that we described in our preliminary study is similar to the magnitude of the benefit produced by several new drugs approved and reimbursed in Italy during the last few years. ${ }^{19}$ Therefore, the negative effect of FT, when occurring, may ultimately lessen the positive impact of some anticancer drugs; on the contrary, effective strategies contrasting FT, might synergise with anticancer treatment and produce relevant benefit for cancer patients.

\section{METHODS AND ANALYSIS}

The project will be conducted according to the methodology delineated by the International Society for Pharmaco-economics and Outcome Research (ISPOR) Patient Reported Outcomes Content Validity Good Research Practices Task Force. ${ }^{20} 21$

The project includes five tasks, summarised in table 1 .

\section{Task 1: concept elicitation and coding}

Specific aim

To elicit concepts and coding them into a thematic library.

\section{Context of use}

Our preliminary data were obtained in a context of Medical Oncology units in public general hospitals, University Oncology units and public Cancer Institutes in Italy, where patients with specific types of solid tumours (lung, breast or ovarian cancer), mostly at an advanced stage of disease, underwent medical treatment, prevalently first-line therapy. In this project, the context will be expanded in order to include patients with any type of solid cancer or haematological malignancy who are undergoing or have recently completed medical treatment, including chemotherapy, target agents, immunotherapy, hormonal treatment, radiotherapy and combinations of such therapies.

\section{Inclusion and exclusion criteria}

The following are the major inclusion/exclusion criteria of the patients who will be involved in the project, also representing the target population for the use of the instrument in future studies.

\section{Inclusion criteria}

- Adult patients (>18 years).

- Histologically or cytologically confirmed diagnosis of any type of solid cancer or haematological malignancy. 
Table 1 Summary table of planned tasks

\begin{tabular}{|c|c|c|c|c|c|}
\hline \multirow[b]{2}{*}{ Title } & \multirow{2}{*}{$\begin{array}{l}\text { Task } 1 \\
\text { Concept } \\
\text { elicitation and } \\
\text { coding }\end{array}$} & \multirow{2}{*}{$\begin{array}{l}\text { Task } 2 \\
\\
\text { Item generation } \\
\text { and analysis }\end{array}$} & \multirow{2}{*}{$\begin{array}{l}\text { Task } 3 \\
\text { Instrument } \\
\text { refinement and } \\
\text { internal validation }\end{array}$} & \multirow{2}{*}{$\begin{array}{l}\text { Task } 4 \\
\text { External validation }\end{array}$} & \multirow{2}{*}{$\begin{array}{l}\text { Task } 5 \\
\\
\text { Application in } \\
\text { clinical studies }\end{array}$} \\
\hline & & & & & \\
\hline Aim & $\begin{array}{l}\text { To elicit concepts } \\
\text { and coding them } \\
\text { into a thematic } \\
\text { library. }\end{array}$ & $\begin{array}{l}\text { To generate a } \\
\text { draft version of } \\
\text { the instrument. }\end{array}$ & $\begin{array}{l}\text { To generate the } \\
\text { final version of the } \\
\text { instrument. }\end{array}$ & $\begin{array}{l}\text { To generate data on } \\
\text { external validity of } \\
\text { the final instrument. }\end{array}$ & $\begin{array}{l}\text { Use in prospective } \\
\text { clinical studies. }\end{array}$ \\
\hline $\begin{array}{l}\text { Psychologists } \\
\text { involved }\end{array}$ & $\begin{array}{l}\text { Yes, for focus } \\
\text { group and coding } \\
\text { activities. }\end{array}$ & $\begin{array}{l}\text { Yes, for cognitive } \\
\text { interviews and } \\
\text { analysis. }\end{array}$ & No & No & No \\
\hline Patients involved & $\begin{array}{l}30 \text { divided in three } \\
\text { focus groups and } \\
\text { approximately } \\
25 \text { for qualitative } \\
\text { interviews. }\end{array}$ & $\begin{array}{l}90 \text { ( } 45 \text { for } \\
\text { importance } \\
\text { analysis and } \\
45 \text { for cognitive } \\
\text { interviews). }\end{array}$ & $\begin{array}{l}118 \text { based on sample } \\
\text { size estimation. }\end{array}$ & $\begin{array}{l}220 \text { based on sample } \\
\text { size estimation. }\end{array}$ & $\begin{array}{l}1000 \text { based on } \\
\text { gross estimation. }\end{array}$ \\
\hline Duration & 6 months & 9 months & 9 months & 12 months & 24 months \\
\hline
\end{tabular}

FT, financial toxicity; HADS, Hospital Anxiety and Depression Scale.

- Written informed consent provided.

- Medical treatment (chemotherapy, target agents, immunotherapy, hormonal treatment, radiotherapy or combinations of such therapies) ongoing or terminated within the previous 3 months.

- Caregivers of patients who meet the above criteria.

\section{Exclusion criteria}

- Patients with major cognitive dysfunction or psychiatric disorders.

- Patients who have never received anticancer medical or radiation treatment.

Sources for concept elicitation

Four different sources of information will be used for concept elicitation.

\section{Literature review}

A literature review will be conducted including international and Italian documents including scientific documents found through PubMed, CINAHL and EMBASE by cross checking references, and publicly available documents not cited in PubMed but found through patients' advocacy websites, government agencies and common web research engines. Content will be divided in single items that will be subsequently categorised according to the conceptual framework.

\section{Focus groups}

Focus groups will be carried out in three towns, distributed in North, Central and South Italy. Two focus groups will be organised in each town: one with patients receiving 
or having recently received medical anticancer treatment, and one with caregivers of patients receiving or who have recently received anticancer treatment. A maximum of 10 individuals will be included, led by a skilled moderator with the help of an assistant moderator. ${ }^{22}$

All focus groups will be audio-recorded and transcribed verbatim. Content analysis will be conducted with a qualitative software (T-Lab). Qualitative analysis of focus group outcomes will be done using the constant comparison technique guided by the Grounded Theory approach. ${ }^{23} 24$

\section{Expert opinions}

Expert opinions will be sought through the cooperation with Associazione Italiana di Oncologia Medica, Collegio Italiano dei Primari di Oncologia Medica Ospedalieri, Federation of Italian Cooperative Oncology Groups (FICOG), Associazione Italiana Infermieri di Area Oncologica, using web or email surveys.

\section{Interviews}

Two types of interviews will be conducted. Patients and caregivers presenting at the info-points of Italian Association of Cancer Patients (AIMAC) in Italian hospitals in Northern, Central and Southern Italy will be invited to respond to question 28 of the EORTC QLQ-C30 questionnaire after a brief identification of baseline sociocultural characteristics. Those who reveal some degree of economic problem at question 28 (score $\geq 2$ ), will be invited to participate in a brief semistructured interview to describe individual experiences of financial difficulties during cancer treatment. Sample size will be determined based on findings during ongoing qualitative analysis, in order to guarantee saturation, a point when the collection of new data does not shed any further light on the issue under investigation.

Qualitative interviews of consenting patients and caregivers will be conducted by nurses in three participating centres representing Southern (Naples), Central (Rome) and Northern (Turin) Italy, with the aim of exploring the lived experience of financial difficulties that occur during cancer treatment. The sample size will be defined by the principle of saturation (in order to guarantee the presence of patients and caregivers who have experienced the phenomenon), and to have demographic variability by region, sex, gender and age, when possible. It is estimated that $8-10$ patients and caregivers from each centre will be adequate. Subjects participating in the interviews will have signed informed consent, be at least 18 years of age, have had experience of financial difficulty as a patient who has received cancer treatment, or as caregiver who has experienced financial difficulty while caring for a family member receiving treatment for cancer. Subjects with cognitive impairments or any physical or medical reason (ie, excessive fatigue) that would not permit participation in an interview will be excluded. Patients will be interviewed individually, and in private. Interviews will be audio recorded and later transcribed verbatim.
Coding and interpretation

Concepts collected through the analysis of the different sources will be coded in an iterative process with opportunities for data to be re-examined and reanalysed until no new codes or code groupings are identified and all passages from the transcripts have been assigned one or more codes. Redundancy and overlapping content will be controlled. Content comparison analysis will be also associated with the analysis of a qualitative software (eg, T-Lab), and concepts will be divided into different themes.

Data interpretation will flow upward from content comparison analysis, coding procedure results and data outputs from qualitative software. A quasi-statistics approach will be used to attain simple descriptive counts of categories, levels of consensus/dissent and about response patterns among the focus group members or the professionals responding to surveys and interviewees. ${ }^{22}$

\section{Products and timelines of task 1}

A list of concepts organised in a thematic coding library will be the product of task 1 activities, within 6 months from the beginning of the project. The protocol will be published to encourage debate and prompt cooperation with other European countries.

\section{Task 2: item generation and analysis}

Specific aim

To generate a draft version of the instrument.

\section{Criteria for item generation}

Major criteria informing item generation are based on the prevalent characteristics of PRO instruments already available in Italian language and widely used in oncology (eg, EORTC questionnaires). Major criteria include:

- Item structure using a question/response model (rather than statement/agreement).

- Short questions.

- Simple language (avoiding technical words).

- No jargon.

Recall period

No single recall period is best for all measures or all phenomena. ${ }^{25}$ In the context of PROs, the recall period must correspond to the characteristics of the phenomenon of interest and the purpose of the assessment. Similar to the COST instrument, a 7-day recall period will be initially used.

\section{Wording of items and responses}

The formulation of each new item will respect the wording and the language suggested during the concept elicitation phase in order to maintain a high level of fidelity and transparency. ${ }^{26}$ The response scheme will follow a 4-point Likert scale (not at all/a little/quite a bit/very much). Alternative formats will be explored, if needed.

Items will be reviewed separately by the members of the Steering Committee and a consensus will be sought 
for each item, also checking for redundancy, overlapping content and ambiguous language.

\section{Item importance analysis}

Item importance will be ranked by a sample of 45 patients (15 for each centre, Naples, Rome and Turin, selected with the same inclusion/exclusion criteria reported above). Patients will be asked to score importance of items within each theme by using a 4-point Likert scale (not important at all/a little important/quite a bit important/extremely important). Within each theme, at least three items with the highest rank will be retained for subsequent steps.

\section{Cognitive interviews}

The list of items remaining after importance analysis, will be administered to a separate sample of 45 patients ( 15 for each centre, Naples, Rome and Turin, selected with the same inclusion/exclusion criteria reported above) followed by cognitive interviews conducted by trained psychologists. ${ }^{27}$ The number of patients is based on a convenience sampling inspired by a grounded theory framework. ${ }^{24}$ According to this framework, frequencies are rarely important, as one occurrence of the data is potentially as useful as many in understanding the process behind a topic, therefore, sample size should generally follow the concept of saturation.

Within such semistructured interviews, items will be evaluated for comprehensibility, recall, judgement and response, mapping a standardised feedback evaluation. Also, the structure and format of the instrument will be evaluated for readability, clarity of presentation, ease of administration. Consideration will be given to potential changes in mode of administration, as this study will evaluate the administration via tablet or smart phone. In this phase, criteria to retain, modify or reject items will be also based on missing values, item difficulty, discriminatory ability, comprehensibility and relevance.

\section{Products and timelines of task 2}

A draft instrument will be the product of task 2 and should be available by month 15 . A paper focusing on the qualitative research outcomes of the first phases of the project is planned.

\section{Task 3: instrument refinement and internal validation Specific aim}

To generate the final version of the PROFFIT instrument.

\section{Definition of scoring procedures}

A global score deriving from the rough sum of the response scores at each item might be calculated, representing a continuous numeric measure of FT. However, in the definitive decision regarding scoring procedures, the Steering Committee will consider, in addition to a single composite measure, the production of subscores referring to different aspects of FT identified.

\section{Factor analysis}

To refine the instrument from the draft to the final version, a principal components analysis (explorative factor analysis) will be undertaken with data from the first field test (eigenvalue limit will be set at 1). Structural equation models could also be used to take into account causal variables.

\section{Reliability}

Internal reliability of multi-item scales will be assessed by analysing inter-item correlations and Cronbach's $\alpha$ coefficient adjusted by the number of items. Estimates of $\alpha>0.70$ will be considered acceptable, CIs will be provided by bootstrap methods.

To assess the test-retest reliability, the intraclass correlation coefficient (ICC) will be estimated repeating the questionnaire administration within a week from the first use. With one-sided alpha $0.05,80 \%$ power, a minimally acceptable level of reliability equal to 0.70 and an expected ICC of $0.80,118$ patients are needed (10-12 for each centre, in 10-12 oncological centres distributed in North, Central and South Italy, selected with the same inclusion/exclusion criteria reported above).$^{28}$

\section{Products and timelines of task 3}

The final instrument will be the product of task 3 and should be available by month 24 . A paper presenting the final instrument is planned.

\section{Task 4: external validation}

Specific aim

To generate data on external validity of the final PROFFIT instrument.

\section{External validation}

Criterion validity will be performed using different anchors. First, the correlation of PROFFIT with response to question 28 of the EORTC QLQ-C30 questionnaire will be assessed. Second, based on the assumption that a high degree of financial problems correlates with worse QoL, the correlation with the global health-related QoL EORTC score (questions 29-30) will be assessed. A similar analysis done with the COST score showed a Pearson correlation coefficient of $0.26 .{ }^{16}$ We plan to test correlations with bilateral alpha $0.05,80 \%$ power and expected Pearson correlation coefficient of 0.20 . For this analysis, 194 patients are required and around 220 patients will be enrolled to allow for some missing data. Descriptively, correlation of PROFFIT with the Hospital Anxiety and Depression Scale (HADS) will be studied as well. For this analysis, 20 patients will be enrolled for each centre, in 10-12 oncological centres distributed in North, Central and South Italy; selection will be based on the same inclusion/exclusion criteria reported above.

\section{Responsiveness}

To test responsiveness (to which extent the instrument is sensitive in capturing the changes of the variable of interest within-patients) repeated measures (at each cycle 
End-point model 1. Interventional, to reduce financial toxicity (FT)

\begin{tabular}{|ll|}
\hline Concept & Endpoints \\
Intervention & Primary \\
$\begin{array}{l}\text { Modifying conditions that } \\
\text { may cause FT }\end{array}$ & FT score (proFFiT) \\
& \\
Supportive concept & Secondary \\
Management efficacy & Survival \\
outcomes & QoL \\
& Toxicity \\
\hline
\end{tabular}

End-point model 2. Interventional, to test efficacy of new treatments

\begin{tabular}{|ll|}
\hline Concept & Endpoints \\
Intervention & Primary \\
$\begin{array}{l}\text { Comparison of new vs } \\
\text { standard treatment }\end{array}$ & Survival \\
& Other time outcomes \\
Supportive concept & Secondary \\
Effect on other efficacy/activity & QoL \\
measure & Toxicity \\
& Response rate \\
& FT score (proFFiT) \\
\hline
\end{tabular}

End-point model 3. Observational, cross sectional, describing FT

\begin{tabular}{|ll|}
\hline Concept & Endpoints \\
Intervention & Primary \\
Describing FT & FT score (proFFiT) \\
& \\
Supportive concept & Secondary \\
Anchors & Socioeconomical condition \\
& QoL \\
& Survival \\
\hline
\end{tabular}

Figure 1 Endpoint models for future use of PROFFIT. PROFFIT, Patient Reported Outcome for Flghting Financial Toxicity of cancer; QoL, quality of life.

or every 3-4 weeks) of PROFFIT will be collected together with the EORTC QLQ-C30. ${ }^{29}$ Changes over time will be estimated. Further correlation of PROFFIT with response to question 28 will be evaluated. Responsiveness will be longitudinally tested with the same sample of patients planned for criterion validity.

\section{Products and timelines of task 4}

Description of correlation of the final instrument with external anchors will be the product of task 4 and should be available by month 36. A paper will be submitted reporting the external validation data.

\section{Task 5: application in clinical studies}

\section{Specific aim}

Use of PROFFIT in subsequent prospective patient series is the aim of task 5. This task is reported here for completeness. Specific protocols will be developed and will undergo proper approval once tasks 1-4 have been completed.

\section{Endpoint models}

PROFFIT may be used in at least three different endpoint models, depicted in figure 1.

\section{Clinical study within the endpoint model 1}

A large-scale multicentre cross-sectional observational study will be performed, where PROFFIT will be used to describe FT across a patient population defined according to type or stage of disease, or any other selection criteria.

The study will include patients with any type of solid tumours or haematological malignancy.

Specific aims will be:

- To describe prevalence of FT overall, and according to different subcategories (geographical, personal, socioeconomical, cancer-related, treatment-related).

- To describe the behaviour of PROFFIT within repeated measures over the time in patients undergoing medical treatment for cancer.

- To describe variability of psychometric properties of PROFFIT over the time.

- To describe the correlation between PROFFIT and typical endpoint of cancer treatments (eg, compliance, QoL, survival).

The study will include at least 1000 patients and strategies will be undertaken to include relevant categories in terms of gender, age, geographic location, type of cancer, performance status and type of treatment (classes of drugs, radiotherapy). During this phase, the cooperation of major cooperative Italian groups (represented by the FICOG) will be of primary importance because it will guarantee feasibility with many participating centres.

\section{Clinical study within the endpoint model 2}

Endpoint model 2, studies might be proposed based on the content of PROFFIT instrument. According to the items that will be retained in the final instrument, we might be faced with different material conditions that may be cause of financial distress. All the efforts will be done to understand how such conditions can be experimentally modified and prospective pilot studies will be planned to produce preliminary evidence on the possible efficacy of corrective actions. The number and the types of these trials will depend on the type of material conditions listed in the PROFFIT.

\section{Clinical study within the endpoint model 3}

The planned use of the instrument in the endpoint model 3 studies is more traditional. The instrument may be used as one of the elements analysed as secondary endpoint in trials testing the efficacy of new drugs or treatment strategies. In principle, consideration should always be given to the context of development of the instrument and therefore this model would apply to the Italian context only. Such studies might be implemented within this project, either based on the findings from previous phases or planned by other independent investigators, once PROFFIT has been made available to the Italian scientific community. 


\section{Data collection procedures}

The Unità Sperimentazioni Cliniche at Istituto Nazionale per lo Studio e la Cura dei Tumori Fondazione G. Pascale IRCCS manages a website (www.usc-intnapoli.net) hub platform for the conduction of multicentre clinical trials, including facilities for patients registration, randomisation, data collection through electronic case report forms, data management through an e-query system, and collection of radiological examination for independent review. Recently, the collection of PRO questionnaires from mobile devices has been implemented and will be used in this project. All website activities are fully tracked and verifiable and the whole system is under a quality assurance programme. The site is accessible by username and password, and is protected through encrypted data certified by SSL and HTTPS protocols. From 2007 to 2015, 180000 web accesses have produced 216000 electronic case report forms. Data are managed by an in-house server, with regular backup, redundant features and onsite maintenance.

Therefore, patient registration and data collection will be centralised and web-based. Interviews, audio-visual data management will be analysed and transferred for storage by a dedicated software.

\section{Patient and public involvement}

The Steering committee of the protocol includes four representatives (FDL, LDC, EI, FT) of Italian and European patients' associations (FAVO, AIMAC, ECPC) who meet regularly with all the other members and share all the decisions assumed by the committee. Therefore, they partnered for the design of the study, some of the activities performed in the concept elicitation phase, and in all the steps where the Steering Committee will assume decisions regarding the project. They will also be involved in dissemination and proposition of future studies using the PROFFIT instrument.

\section{Project status}

March 2018: start of tasks including patients (focus groups).

October 2019: planned time for submission of a paper reporting on qualitative tasks.

March 2020: planned time for final instrument availability.

March 2021: planned time for submission of a paper reporting on the final validated instrument (end of task 4).

\footnotetext{
Author affiliations

${ }^{1}$ Department of Psychology and Pedagogical Science, Faculty of Sport, Health and Applied Science, St.Mary's University, Twickenham, London, UK

2Dipartimento di Medicina Interna, IRCCS Ca' Granda Foundation, Università di Milano, Milano, Italy

${ }^{3}$ Unità Sperimentazioni Cliniche, Istituto Nazionale per lo Studio e la Cura dei Tumori, IRCCS Fondazione Pascale, Napoli, Italy

${ }^{4}$ St. John Clinical Research Institute, St. John Medical Center, Tulsa, Oklahoma, USA ${ }^{5}$ Departmento di Biomedicina e Prevenzione, Università degli Studi di Roma Tor Vergata, Roma, Italy

${ }^{6}$ Associazione Italiana Malati di Cancro (AIMAC), Roma, Italy
}

${ }^{7}$ European Cancer Patient Coalition (ECPC), Brussels, Belgium

${ }^{8}$ Federazione italiana delle Associazioni di Volontariato in Oncologia (FAVO), Roma, Italy

${ }^{9}$ Dipartimento di Oncologia, A0 Ordine Mauriziano, Università degli Studi di Torino, Torino, Italy

${ }^{10}$ Health Outcomes Research Unit, Fondazione GIMEMA, Roma, Italy

${ }^{11}$ Federation of Italian Cooperative Oncology Groups (FICOG), Milano, Italy

${ }^{12}$ Unità di Biostatistica, Istituto Nazionale per lo Studio e la Cura dei Tumori Regina

Elena, IRCCS, Roma, Italy

${ }^{13}$ Dipartimento di Economia, Università degli Studi di Messina, Messina, Italy

${ }^{14}$ Practice, Government, Health and Not for Profit Division, SDA Bocconi School of Management, Milano, Italy

${ }^{15}$ Oncologia, Azienda Ospedaliera Specialistica dei Colli, Napoli, Italy

${ }^{16}$ Area Welfare e Salute, Censis - Centro Studi Investimenti Sociali, Roma, Italy

${ }^{17}$ Statistica Medica, Università degli Studi della Campania Luigi Vanvitelli, Napoli, Italy

Acknowledgements The authors thank Lucia Sparavigna, Anna Gimigliano, Giuliana Canzanella, Laura Arenare, Fiorella Romano and Alfonso Savio for their contribution to project management. They also thank, for their contribution in patients' management, Francesca Laudato, Maria Rosaria Esposito, Ermelinda Quarata (Napoli), Sabrina Terzolo, Luisa Fusco, Annalisa Bellezza, Laura Polimeno (Torino) and Filomena Spasiano, Luana Fotia (Roma).

Contributors FP obtained funding. SR, JB, CG and FP planned qualitative and statistical analysis and drafted the protocol. All authors (SR, JB, FDL, LDC, MDM, FE, LF, DG, LG, El, CJ, VM, FT, CMV, CG, FP) participated in writing the study protocol and the manuscript and approved the final version.

Funding The project is supported by Fondazione AIRC (Associazione Italiana per la Ricerca sul Cancro), a non-profit Italian charity, IG 2017 Id 20402.

Competing interests SR has received personal fees from CSL-Behring and GlaxoSmithKline Foundation. JB has received personal fees from Novartis, AstraZeneca, Merck Sharp \& Dohme. MDM has received personal fees from Bristol Myers Squibb, Merck Sharp \& Dohme, Roche, AstraZeneca, Janssen. FE has received personal fees from Bristol Myers Squibb, Incyte, Orsenyx and Amgen. LG has received personal fees from Bristol Myers Squibb. CJ has received personal fees from Amgen, Astra Zeneca, Biogen, Boehringer Ingelheim, Celgene, Gilead, GSK, Ipsen, Janssen-Cilag, Takeda and Sanofi. VM has received personal fees from Bristol Myers Squibb and Italfarmaco; a member of his family is employee in Bayer. CMV has received personal fees from Baxter, MSD, Novartis, Sanofi, Sanofi Genzyme. FP has received personal fees from Bayer, Ipsen, Astra Zeneca, Bristol Myers Squibb, Sandoz, Incyte, Celgene, Pierre Fabre, Janssen-Cilag.

Patient consent for publication Not required.

Ethics approval By 6 May 2019, the protocol has been approved by Ethical Committees at all centres participating in the first two tasks of the protocol.

Provenance and peer review Not commissioned; externally peer reviewed.

Open access This is an open access article distributed in accordance with the Creative Commons Attribution Non Commercial (CC BY-NC 4.0) license, which permits others to distribute, remix, adapt, build upon this work non-commercially, and license their derivative works on different terms, provided the original work is properly cited, appropriate credit is given, any changes made indicated, and the use is non-commercial. See: http://creativecommons.org/licenses/by-nc/4.0/.

\section{REFERENCES}

1. Mailankody S, Prasad V. Five years of cancer drug approvals: innovation, efficacy, and costs. JAMA Oncol 2015;1:539-40.

2. Saltz LB. Perspectives on cost and value in cancer care. JAMA Oncol 2016;2:19-21.

3. Ramsey S, Blough D, Kirchhoff A, et al. Washington state cancer patients found to be at greater risk for bankruptcy than people without a cancer diagnosis. Health Aff 2013;32:1143-52.

4. Chino F, Peppercorn J, Taylor DH, et al. Self-Reported financial burden and satisfaction with care among patients with cancer. Oncologist 2014;19:414-20.

5. Zafar SY, Peppercorn JM, Schrag D, et al. The financial toxicity of cancer treatment: a pilot study assessing out-of-pocket expenses and the insured cancer patient's experience. Oncologist 2013;18:381-90. 
6. Lathan CS, Cronin A, Tucker-Seeley R, et al. Association of financial strain with symptom burden and quality of life for patients with lung or colorectal cancer. J Clin Oncol 2016;34:1732-40.

7. Zafar SY, McNeil RB, Thomas CM, et al. Population-Based assessment of cancer survivors' financial burden and quality of life: a prospective cohort study. JOP 2015;11:145-50.

8. Delgado-Guay M, Ferrer J, Rieber AG, et al. Financial distress and its associations with physical and emotional symptoms and quality of life among advanced cancer patients. Oncologist 2015;20:1092-8.

9. Ramsey SD, Bansal A, Fedorenko CR, et al. Financial Insolvency as a risk factor for early mortality among patients with cancer. Journal of Clinical Oncology 2016;34:980-6.

10. Büttner M, König H-H, Löbner M, et al. Out-of-pocket-payments and the financial burden of 502 cancer patients of working age in Germany: results from a longitudinal study. Support Care Cancer 2019;27:2221-8.

11. Ezeife DA, Morganstein BJ, Lau S, et al. Financial burden among patients with lung cancer in a Publically funded health care system. Clin Lung Cancer 2019;20:231-236.

12. Honda K, Gyawali B, Ando M, et al. A prospective survey of comprehensive score for financial toxicity in Japanese cancer patients: report on a pilot study. Ecancermedicalscience 2018;12:847.

13. Newton JC, Johnson CE, Hohnen H, et al. Out-Of-Pocket expenses experienced by rural Western Australians diagnosed with cancer. Support Care Cancer 2018;26:3543-52.

14. de Souza JA, Yap BJ, Hlubocky FJ, et al. The development of a financial toxicity patient-reported outcome in cancer: the cost measure. Cancer 2014;120:3245-53.

15. Altice CK, Banegas MP, Tucker-Seeley RD, et al. Financial Hardships experienced by cancer survivors: a systematic review. J Natl Cancer Inst 2016.

16. de Souza JA, Yap BJ, Wroblewski K, et al. Measuring financial toxicity as a clinically relevant patient-reported outcome: the validation of the comprehensive score for financial toxicity (cost). Cancer 2017;123:476-84.

17. Honda K, Gyawali B, Ando M, et al. Prospective survey of financial toxicity measured by the comprehensive score for financial toxicity in Japanese patients with cancer. J Glob Oncol 2019;5:1-8.
18. Scott F Huntington BMW, DAN T Vogl, ADAM D Cohen, Alfred L Garfall, Patricia a Mangan, Jalpa a Doshi, Edward a Stadtmauer. financial toxicity in insured patients with multiple myeloma: a crosssectional pilot study. Lancet Haematol 2015;2:e408-16.

19. Perrone F, Jommi C, Di Maio M, et al. The association of financial difficulties with clinical outcomes in cancer patients: secondary analysis of 16 academic prospective clinical trials conducted in Italy. Annals of Oncology 2016;27:2224-9.

20. Patrick DL, Burke LB, Gwaltney CJ, et al. Content ValidityEstablishing and reporting the evidence in newly developed patient-reported outcomes (pro) instruments for medical product evaluation: ISPOR pro good research practices Task force report: part 2-Assessing Respondent understanding. Value in Health 2011;14:978-88.

21. Patrick DL, Burke LB, Gwaltney CJ, et al. Content validity-establishing and reporting the evidence in newly developed patient-reported outcomes (PRO) instruments for medical product evaluation: ISPOR PRO good research practices task force report: part 1--eliciting concepts for a new PRO instrument. Value Health 2011;14:967-77.

22. Onwuegbuzie AJ, Dickinson WB, Leech NL, et al. A qualitative framework for collecting and analyzing data in focus group research. Int J Qual Methods 2009;8:1-21.

23. Glaser BG. Theoretical sensitivity. Mill Valley, CA: Sociology Press, 1978.

24. Glaser BG. Discovery of grounded theory. Chicago, IL: Aldine, 1992

25. Stull DE, Leidy NK, Parasuraman B, et al. Optimal recall periods for patient-reported outcomes: challenges and potential solutions. Curr Med Res Opin 2009;25:929-42.

26. Heritage J. Conversation analysis and institutional talk: Analyzing data. In: Silverman D, ed. Qualitative research: theory, method, and practice. Thousand Oaks, CA: Sage, 2004: 222-45.

27. Onwuegbuzie AJ. Typology of mixed methods sampling designs in social science research. The Qualitative Report 2007;12:281-316.

28. Walter SD, Eliasziw M, Donner A. Sample size and optimal designs for reliability studies. Stat Med 1998;17:101-10.

29. Aaronson NK, Ahmedzai S, Bergman B, et al. The European organization for research and treatment of cancer QLQ-C30: a quality-of-life instrument for use in international clinical trials in oncology. J Natl Cancer Inst 1993;85:365-76. 